\title{
Virtual Tenant Network Research based on Price Mechanism in SDN Architecture
}

\author{
Baoxia Wang ${ }^{a}$, Zhuge Bin ${ }^{b}$, Minhui Yao ${ }^{c}$ and Weiming Wang ${ }^{d}$ \\ Institute of Networks and Communications Engineering, Zhejiang Gongshang University, Hangzhou \\ 310018, China \\ aabile0824@126.com, b'zhugebin@mail.zjgsu.edu.cn, cyaominhui1989@163.com, dwmwang@mail. \\ zjgsu.edu.cn
}

Keywords: Software Defined Networking, price mechanism, resource, Virtual Tenant Network.

\begin{abstract}
Software-Defined Networking (SDN) is a hot transforming networking architecture and technology at present. In the SDN architecture, the network device control and data plane are decoupled to achieve the segregation of the control and forwarding. The SDN architecture opens the REST APIs so that the developers can call them. This paper surveys the price mechanism in the Open Daylight VTN. We design the generic price negotiation module and the generic trade module, and each layer can call them for pricing resources. Therefore, this mechanism can provides a kind of price strategy in order to choose a more appropriate route to the user.
\end{abstract}

\section{Introduction}

Software Defined Networking (SDN) is a new network architecture evolved from at Stanford University clean slate research group and UC Berkeley around 2008, its core technology Open Flow mainly has three functions: separation of the control and data planes; using a standardized protocol between controller and an agent on the network element for instantiating state; providing network programmability from a centralized view via a modern, extensible API[1,2,3,5].So it can provide a good platform for the innovation of core networks and applications.

A few recent papers have explored the technology_-Virtual Tenant Network (VTN), which is one of the Open Daylight projects, mainly provides a kind of virtual network to users in Open Daylight open projects. As the development of Open Daylight, the application VTN will also be widely used, but the lack of price mechanism will impede its smooth development of VTN [6]. With the granularity of pricing tinier, not only the resource can be priced, but also the function in the procedure can be done.

In this paper, we want to present a kind of price mechanism added to the VTN project. It makes users select VTN depending on price and required resources.

\section{VTN Technology}

Currently, the mainstream SDN architecture framework is mainly made of the SDN architecture proposed by the Open Networking Foundation (ONF), the Network Functions Virtualization (NFV) architecture proposed by European Telecommunications Standards Institute (ETSI) and Open Daylight open source projects [4]. Open Daylight run in the support of Linux Foundation, and it aims to provide an open platform for Software Defined Networks and Network Functions Virtualization, so that any manufacturers can make use of the open platform. The sub-project Open Daylight Virtual Tenant Network (VTN) is an application that provides multi-tenant virtual network on an SDN controller [7].Due to the multi-tenant virtual network with resource sharing in the same physical network, different tenants can configure different applications and run different network according to the needs.

VTN includes two parts: VTN Coordinator and VTN Manager, and VTN Coordinator locate in the network applications orchestrations \& services, while VTN Manager lies into the controller platform. 
The unique feature of VTN is that it has a logical abstraction plane, which can completely separates the logical plane from the physical plane. Hence users can design and arrange any networks they wanted without understanding any configuration in the physical plane. VTN allows the users to deploy the L2/L3 network. Once the network is deployed, the technology mapping will automatically be used, which can make the network map into the underlying physical network. Any VTN will be isolated, but they have the same resources based on the same physical network. VTN makes the management easily and hides the complexity of the physical network.

\section{The Price Mechanism based on VTN}

The end-to-end links in physical layer have many links, while each link has different cost, when the user establishes the VTN. This paper intends to design a pricing mechanism based on VTN, in which the user can proposes the appropriate VTN price, according to the resources they need to match an appropriate VTN. The VTN internal mechanisms from coordinator to manager and then to the underlying resources, various parts of the module call the generic prices module, and implement the real-time pricing for resources. Each layer negotiates with the resources' price, and ultimately to the user after the price has been negotiated and the corresponding VTN chain road. The major steps is according to the pricing algorithm when Implementing the real-time pricing of resources, and then the subsequent pricing is based on the frequency of using the VTN, the price from the user and the shortage of resources .

This paper mainly talks about designing a generic price module in the VTN architecture, including the design of the generic price negotiation module and the generic trade module.

The generic price module is located into every layer of the SDN architecture, it can provide a framework in which using price algorithm. The developer can put their designed price algorithm into this framework, so that can achieve the price mechanism in SDN.

The design of the generic price negotiation module (SDP). Generally speaking, the generic price negotiation module lies between the data plane elements and the controller platform, also between the controller platform and the network applications orchestrations \& services. In the process of selecting and negotiating using needs and budget constraints, the goal is to get the most optimal level of service quality under the service consumer determines the budget constraints.

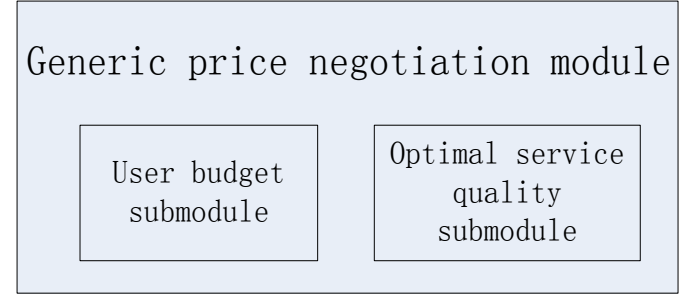

Fig. 1 The generic price negotiation module

The generic price negotiation module principally contains the user budget submodule and the optimal service quality submodule. It provides a generic interface-Price Negotiation, which including two methods: Public Double budget () \{\} and public Map $<$ String, Object $>$ resource () \{\}.

Any module needed the price mechanism should implement the interface, budget () method is to provide a user-budget algorithm for the developers, while resources () method is primarily designed for having rational resources to the developers.

Meanwhile, generic price negotiation module includes auto-negotiation mechanism, with auto-negotiation mechanism, the application layer can put forward their own requirements according to the budget, and then the controller layer can get the optimal resource services based on the user's budget. If it cannot meet the needs from the user, then the process of negotiating fails; on the contrary, the negotiation is successful.

The design of the Generic Trade Module. The Generic Trade Module is primarily responsible for the pricing of resources, user's transaction and balance. It mainly consists of Virtual Resource submodule, Expenditure submodule, Pricing submodule, Balance submodule, Account submodule (see Fig.2). 


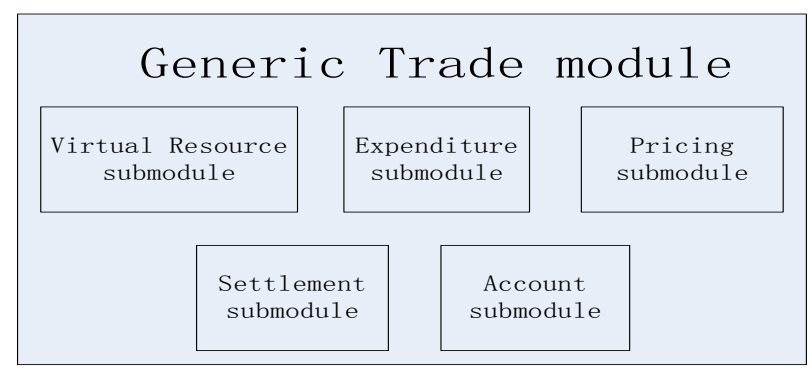

Fig. 2 The generic trade module

Virtual Resource submodule: include the underlying physical resources, all behavior of the users; Include the changes of daily traffic, all of these records will be stored into the database.

Expenditure submodule: include the user's consumption behavior and trade behavior.

Pricing submodule: analyze the distribution of the flow in each time interval and each link through the long time user's behaviors, and then determine the price of that resources used by users.

Settlement submodule: be used to settle an account for the user's expenditure.

Account submodule: include the user's account, password, balance and the identification to the users.

The Generic Trade module provides a generic interface-Common Price, including two methods: public Double get Price () \{\} and public void algorithm () \{\}. The method get Price () is responsible for returning a price for every resource, while the method algorithm () offers a method of achieving any algorithm.

\section{The Design of VTN system based on Price}

VTN includes VTN Manager in SDN controller and VTN Coordinator in application layer, it is able to provide multi-tenant virtual network for SDN, also request install a variety of network applications for each tenant.

The main function is the implement of Software Defined Price (SDP) mechanism in the Virtual Tenant Network based on price. The SDP mechanism exist each module of VTN, whose primary function is achieving a protocol to negotiate the price and helping the resource have a reasonable price.

Figure 3 depicts the component of VTN based on price.

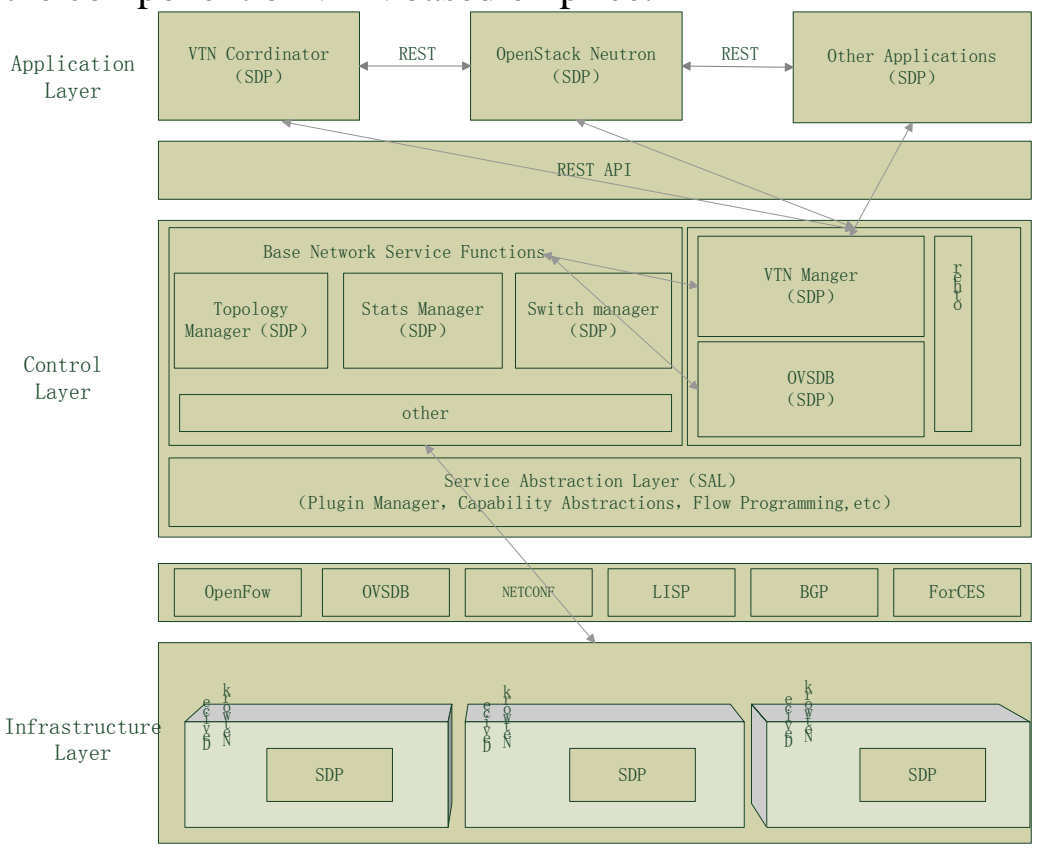

Fig. 3 the architecture of VTN system based on price

From this picture, we know that the VTN system based on price has many modules including the price negotiation and trading mechanism. Firstly, between the Infrastructure Layer and the Control Layer, the Control Layer can get underlying resources’ price through the price negotiation with the 
Infrastructure Layer. Secondly the Controller Layer negotiates the price with the Infrastructure Layer, according to this price Base Network Service Functions will determine their service price with the process successes. Finally, the VTN Manager can through price negotiation get resources' price from the Base Network Service Functions and give its own resources' price on the account of pricing mechanism.

Application calls that needed resources and pays corresponding price via REST API, meanwhile inter-application also do the same things through REST API.

\section{Summary}

In this paper, we want to design a sort of price mechanism in Open Daylight VTN, which can choose different links according to different prices. Further works will be researched in how to achieve those modules and edit code.

\section{Acknowledgments}

This work was supported in part by a grant from the National Basic Research Program of China (973 Program) (No.2012CB315902), the National Natural Science Foundation of China (No.61379120, 61170215), Zhejiang Leading Team of Science and Technology Innovation, Zhejiang Provincial Key (No.2011R50010-9, .2011R50010-11, .2011R50010-14). Laboratory of New Network Standards and Technologies (NNST)(No.2013E10012),The Youth Foundation of Zhejiang Gongshang university(QZ13-8).

\section{References}

[1]Software-Defined Networking: The New Norm for Networks. White paper. Open Networking Foundation. April 13, 2012. Retrieved August 22, 2013.

[2]M.K.Shin, K.H.Nam, H.J.Kim. Software-Defined Networking (SDN): A Reference Architecture and Open APIs[C]//ICT Convergence (ICTC), 2012 International Conference on. IEEE, 2012: 360-361.

[3] Thomas Neadeau and Ken Gray. SDN: Software Defined Networks—an Authoritative Review of Network Programmability Technologies [M]. California: O’Reilly Media, August 2013:384.

[4]B.H. Lei, F. Wang, Q. Wang \& H.Y. Wang. Deciphering SDN: Core Techniques and Practical Guide [M]. Beijing: Publishing House of Electronics Industry, 2013:244.

[5]OpenNetworkingFoundation[EB/OL].[2014-9-15].https://www.opennetworking.org/sdn-resourc es/sdn-definition

[6] Open Daylight[EB/OL].[2014-9-15].http://www.opendaylight.org

[7] VTN[EB/OL]. [2014-9-15]. https://wiki.opendaylight.org/view/Release/Hydrogen/VTN/ User_ Guide 